\title{
QUALIDADE PÓS-COLHEITA DE QUIVI 'HAYWARD' TRATADO COM 1-MCP E ARMAZENADO SOB DIFERENTES ATMOSFERAS ${ }^{1}$
}

\author{
MARCELO JOSÉ VIEIRA², LUIZ CARLOS ARGENTA ${ }^{3}$ \\ CASSANDRO VIDAL TALAMINI DO AMARANTE ${ }^{4}$, \\ AMANDA MARIA FURTADO DREHMER VIEIRA ${ }^{5}$, CRISTIANO ANDRÉ STEFFENS ${ }^{6}$
}

RESUMO - Este trabalho teve por objetivo avaliar o efeito do 1-MCP sobre o amadurecimento e a qualidade pós-colheita de quivi 'Hayward' armazenado sob diferentes atmosferas. Foram conduzidos dois experimentos, com frutos colhidos em pomar comercial localizado em Fraiburgo-SC. No experimento 1, os frutos foram tratados com três doses de 1-MCP $\left(0,0 ; 0,5\right.$ ou $\left.1,0 \mu \mathrm{L} \mathrm{L}^{-1}\right)$ e armazenados sob atmosfera controlada (AC, contendo 2,0kPa de $\mathrm{O}_{2}+4,5 \mathrm{kPa}$ de $\mathrm{CO}_{2}$, a $0^{\circ} \mathrm{C} \pm 0,5^{\circ} \mathrm{C}, 92 \pm 3 \% \mathrm{UR}$, e com $<40 \eta \mathrm{L} \mathrm{L}^{-1}$ de etileno no ambiente), durante 90 e 120 dias, mais sete dias de vida de prateleira a $23^{\circ} \mathrm{C}$. No experimento 2 , os frutos foram tratados com 0,0 ou $0,7 \mu \mathrm{L} \mathrm{L}^{-1}$ de 1-MCP e armazenados sob atmosfera ambiente (AA, contendo 21 $\mathrm{kPa}$ de $\mathrm{O}_{2}+0,03 \mathrm{kPa}$ de $\mathrm{CO}_{2}$, a $0 \pm 0,5^{\circ} \mathrm{C}, 90 \pm 3 \% \mathrm{UR}$, e com 200 a $250 \eta \mathrm{L} \mathrm{L} \mathrm{L}^{-1}$ de etileno no ambiente, durante $30,60,90$ e 120 dias, mais um e sete dias de vida de prateleira a $23^{\circ} \mathrm{C}$ ) ou $\mathrm{AC}$ (contendo $2,0 \mathrm{kPa}$ de $\mathrm{O}_{2}+4,5 \mathrm{kPa}$ de $\mathrm{CO}_{2}$, a $0^{\circ} \mathrm{C} \pm 0,5^{\circ} \mathrm{C}, 92 \pm 3 \% \mathrm{UR}$, e com $<40 \eta \mathrm{L} \mathrm{L}^{-1}$ de etileno no ambiente, durante 60,90, 120 e 150 dias, mais um e sete dias de vida de prateleira a $23^{\circ} \mathrm{C}$ ). Os benefícios do tratamento com 1-MCP sobre a conservação da qualidade foram observados tanto para frutos armazenados sob AA quanto sob AC. No experimento 1, não houve diferença entre as doses de 1-MCP de 0,5 e $1,0 \mu \mathrm{L} \mathrm{L}^{-1}$ sobre o controle do amadurecimento e manutenção da qualidade dos frutos armazenados em AC. No experimento 2, o tratamento com 1-MCP retardou o aumento na taxa de produção de etileno após remoção de câmara fria. A redução da taxa de produção de etileno nos frutos tratados com 1-MCP foi associada ao aumento na conservação da firmeza da polpa sob AC e AA, e redução na incidência de pericarpo translúcido sob AA. O potencial de armazenamento foi aumentado pelo tratamento com 1-MCP em aproximadamente 60 e 30 dias, nos frutos armazenados sob AA e AC, respectivamente.

Termos para indexação: Actinidia deliciosa, etileno, firmeza de polpa, acidez titulável, polpa translúcida, atmosfera controlada.

\section{POSTHARVEST QUALITY OF 'HAYWARD' KIWIFRUIT TREATED WITH 1-MCP AND STORED UNDER DIFFERENT ATMOSPHERES}

\begin{abstract}
The objective of this study was to assess the effect of 1-MCP on ripening and postharvest quality of 'Hayward' kiwifruit stored under different atmospheres. Two experiments were carried out with fruits harvested in a commercial orchard located in Fraiburgo (State of Santa Catarina, Southern Brazil) in April of 2003 (experiment 1) and April of 2004 (experiment 2). For experiment 1, fruit were treated with different doses of 1-MCP $\left(0.0 ; 0.5\right.$ or $\left.1.0 \mu \mathrm{L} \mathrm{L}^{-1}\right)$ and stored in controlled atmosphere (CA, with $2.0 \mathrm{kPa} \mathrm{O}_{2}$ $+4.5 \mathrm{kPa} \mathrm{CO}$, at $0^{\circ} \mathrm{C} \pm 0.5^{\circ} \mathrm{C} / 92 \pm 3 \% \mathrm{RH}$, and ethylene in the atmosphere $\left.<40 \eta \mathrm{L} \mathrm{L}^{-1}\right)$. For experiment 2, fruit were treated with 0.0 or $0.7 \mu \mathrm{L} \mathrm{L}^{-1}$ of $1-\mathrm{MCP}$ and stored in ambient atmosphere (AA; with $21.0 \mathrm{kPa} \mathrm{O}$ $+0.03 \mathrm{kPaCO}$, at $0 \pm 0.5^{\circ} \mathrm{C} / 90 \pm 3 \% \mathrm{RH}$, and $200-250 \eta \mathrm{L} \mathrm{L}^{-1}$ of ethylene in the atmosphere $)$ or $\mathrm{CA}(2.0 \mathrm{kPa}$ $\mathrm{O}_{2}+4.5 \mathrm{kPaCO}$, at $0^{\circ} \mathrm{C} \pm 0.5^{\circ} \mathrm{C} / 92 \pm 3 \% \mathrm{RH}$, and ethylene in the atmosphere $<40 \mathrm{~nL} \mathrm{~L}^{-1}$ ). For experiment 1 , fruit were evaluated after 90 and 120 days of storage at $0^{\circ} \mathrm{C}$, followed by seven days at $23^{\circ} \mathrm{C}$. For experiment 2, fruit were evaluated after 30,60, 90 and 120 days of storage in AA, and after 60, 90, 120 and 150 days of storage in $\mathrm{CA}$, plus one and seven days at $23^{\circ} \mathrm{C}$. The benefits of $1-\mathrm{MCP}$ treatment on fruit quality preservation were observed for fruit stored in both, AA and AC. For experiment 1, there was no difference between 0.5 and $1.0 \mu \mathrm{L} \mathrm{L}^{-1}$ of $1-\mathrm{MCP}$ on fruit ripening and postharvest quality preservation of kiwifruit stored in CA. For experiment 2, the treatment with 1-MCP delayed the increase of ethylene production rate after removal from cold storage. The reduced ethylene production in fruit treated with 1-MCP delayed the loss of flesh firmness in AC and AA, and reduced the incidence of pericarp translucency in AA. The treatment with 1-MCP increased the storage potential by 60 and 30 days for fruit stored in AA and CA, respectively. Index terms: Actinidia deliciosa, ethylene, pulp firmness, titratable acidity, pericarp translucency, controlled atmosphere.
\end{abstract}

\footnotetext{
${ }^{1}$ (Trabalho 259-11). Recebido em: 07-11-2011. Aceito para publicação em:17-05-2011.

${ }^{2}$ Eng. Agr., M.Sc., Renar Maçãs S/A, Rod. da Maçã, Km 28, Cx. P. 21, CEP 89580-000, Fraiburgo-SC. E-mail: marvieira@gmail.com ${ }^{3}$ Eng. Agr., D.Sc., Pesq. da Epagri, Cx. P. 591, CEP 89500-000, Caçador-SC. E-mail: argenta@epagri.sc.gov.br

${ }^{4}$ Eng. Agr., Ph.D., Professor do Dep ${ }^{\text {to }}$ de Agronomia,CAV/UDESC, CEP 88520-000, Lages-SC. E-mail: amarante@cav.udesc.br ${ }_{5}^{5}$ Eng. Agr., M.Sc., Professora do Dep ${ }^{\text {to }}$ de Biotecnologia, UNOESC, Videira-SC. E-mail: amanda.drehmer@unoesc.edu.br ${ }^{6}$ Eng. Agr., Dr., Professor do Departamento de Agronomia, CAV/UDESC, Lages-SC. E-mail: steffens@cav.udesc.br
} 


\section{INTRODUÇÃO}

A produção brasileira de quivi concentra-se na região Sul, sendo a colheita realizada normalmente entre os meses de abril e maio. A adoção de técnicas para prolongar o período de conservação dos frutos, após a colheita, é essencial para aumentar o período de comercialização (VIEIRA et al., 2010).

$\mathrm{O}$ armazenamento refrigerado sob condição de atmosfera controlada (AC) foi recomendado para a conservação pós-colheita de quivi por VIEIRA et al. (2010). No entanto, pode ocorrer acúmulo de etileno na atmosfera das câmaras de armazenamento, dependendo do ponto de colheita dos frutos e do período de armazenamento. $\mathrm{O}$ etileno é responsável por regular a maturação e a senescência de frutos, pois altera a expressão de genes responsáveis por vários processos fisiológicos, incluindo sua autocatálise, a respiração climatérica e as mudanças da textura, cor, sabor e aroma (WATKINS, 2006). O quivi é considerado o fruto mais sensível à ação do etileno, e exposição a concentrações de etileno inferiores a $10 \eta \mathrm{L} \mathrm{L}^{-1}$ já é suficiente para acelerar significativamente seu amadurecimento (ARPAIA et al., 1994).

O controle do amadurecimento de frutos com o uso do 1-metilciclopropeno (1-MCP), um inibidor da ação do etileno, prolonga a vida pós-colheita dos mesmos (WATKINS, 2006). Este inibidor reduz a produção de etileno e retarda a maturação, estendendo a vida pós-colheita de inúmeras espécies de frutos climatéricos, incluindo tomate, banana, maçã, abacate e pera (BLANKENSHIP; DOLE, 2003).

As respostas ao tratamento com 1-MCP podem ser influenciadas por diversos fatores, dentre os quais a concentração, o tempo de exposição, o tempo entre a colheita dos frutos e a aplicação do produto, a atmosfera de armazenamento e a cultivar (WATKINS, 2006).

Este trabalho teve por objetivo avaliar o efeito do 1-MCP sobre o amadurecimento e a qualidade pós-colheita do quivi 'Hayward' armazenado sob diferentes atmosferas.

\section{MATERIAL E MÉTODOS}

\section{Colheita e armazenamento dos frutos}

Dois experimentos foram conduzidos com frutos colhidos em pomar comercial do município de Fraiburgo-SC, em abril de 2003 (experimento 1) e abril de 2004 (experimento 2). Após a colheita, eles foram selecionados quanto à uniformidade do tamanho $(110 \pm 5 \mathrm{~g})$ e ausência de defeitos.

No experimento 1 , os frutos foram tratados com três doses de 1-MCP $\left(0,0 ; 0,5\right.$ ou $\left.1,0 \mu \mathrm{L} \mathrm{L}^{-1}\right)$ e armazenados por 90 e 120 dias em câmara fria sob atmosfera controlada (AC, $2,0 \mathrm{kPa}$ de $\mathrm{O}_{2}+4,5 \mathrm{kPa}$ de $\mathrm{CO}_{2}$, a $0^{\circ} \mathrm{C} \pm 0,5^{\circ} \mathrm{C}, 92 \pm 3 \% \mathrm{UR}$, e com etileno no ambiente $<40 \eta \mathrm{L} \mathrm{L}^{-1}$ ). No experimento 2, os frutos foram tratados com 0,0 ou $0,7 \mu \mathrm{L} \mathrm{L}^{-1}$ de 1 -MCP e armazenados por 30; 60; 90 e 120 dias em câmara fria sob atmosfera ambiente (AA, $21 \mathrm{kPa}$ de $\mathrm{O}_{2}+0,03 \mathrm{kPa}$ de $\mathrm{CO}_{2}$, a $0,0 \pm 0,5^{\circ} \mathrm{C}, 90 \pm 3 \% \mathrm{UR}$, e com etileno no ambiente de 200 a $250 \eta \mathrm{L} \mathrm{L}^{-1}$ ) ou por $60 ; 90 ; 120$ e 150 dias em câmara fria sob atmosfera controlada (AC, $2,0 \mathrm{kPa}$ de $\mathrm{O}_{2}+4,5 \mathrm{kPa}$ de $\mathrm{CO}_{2}$, a $0^{\circ} \mathrm{C} \pm 0,5^{\circ} \mathrm{C}$, $92 \pm 3 \%$ UR, e com etileno no ambiente $<40 \eta \mathrm{L} \mathrm{L}^{-1}$ ).

$\mathrm{O}$ tratamento com o gás 1-MCP foi realizado em câmara de 340 L, fabricada em PVC e hermeticamente fechada com tampa de acrílico. O gás 1-MCP foi gerado misturando 4 gramas de EthylBloc ${ }^{\mathrm{TM}}$ (AgroFresh Inc., 0,14\%) com água a $25^{\circ} \mathrm{C}$, num frasco de $500 \mathrm{~mL}$, conectado à câmara de tratamento. O gás 1-MCP foi bombeado para a câmara de tratamento em um sistema fechado, durante 15 minutos (tempo necessário para atingir a concentração de interesse). A concentração do gás 1-MCP durante o tratamento foi determinada por cromatografia gasosa, seguindo metodologia descrita por Argenta et al. (2003). Após o tratamento com 1-MCP, os frutos foram armazenados em câmaras comerciais de armazenamento de quivi. Os frutos foram submetidos à condição de $\mathrm{AC}$ um dia após a aplicação do 1-MCP. Para estabelecimento da AC, o gás $\mathrm{O}_{2}$ foi diluído da atmosfera de armazenamento por meio da introdução do gás $\mathrm{N}_{2}$ utilizando o princípio "Pressure Swing Absorption" (PSA) até a pressão parcial de interesse. $\mathrm{O}$ aumento da pressão parcial de $\mathrm{CO}_{2}$ foi obtido naturalmente pela respiração dos frutos. A pressão parcial dos gases $\mathrm{O}_{2}$ e $\mathrm{CO}_{2}$ foi monitorada diariamente, a cada 2 horas, utilizando analisadores marca Agri-Datalog Modelo ST-CONTROL 2000. $\mathrm{O} \mathrm{O}_{2}$ consumido pela respiração foi compensado por meio da introdução de ar nas câmaras. Adsorvedores marca Agri-Datalog Modelo AK 2/300 foram utilizados para manter as pressões parciais de $\mathrm{CO}_{2}$ dentro dos limites estabelecidos. Apenas na câmara de AC, um conversor de etileno (marca Agri-Datalog Modelo Katox 600) permaneceu acionado para contínua remoção do etileno. Periodicamente, amostras de ar foram coletadas para determinar o etileno no ambiente de armazenamento, o qual variou de 200 a $250 \eta \mathrm{L} \mathrm{L}^{-1}$ sob AA, e de 10 a $40 \eta \mathrm{L} \mathrm{L}^{-1}$ sob AC.

\section{Análise dos frutos}

A qualidade dos frutos foi avaliada na colheita e após cada período de armazenamento indicado para as condições de $\mathrm{AA} \mathrm{e} \mathrm{AC}$, mais 7 dias a $23^{\circ} \mathrm{C}$. Durante o período de prateleira $\left(7\right.$ dias a $\left.23^{\circ} \mathrm{C}\right)$, os frutos ficaram expostos a $1,0 \mu \mathrm{L} \mathrm{L}^{-1}$ de etileno. Os frutos foram analisados quanto à firmeza da polpa, teor de sólidos solúveis (SS), acidez titulável (AT) e ocorrência de pericarpo translúcido e podridões.

A análise da firmeza da polpa foi realizada em um lado da superfície de cada fruto, utilizando-se de um penetrômetro com ponteira de $8 \mathrm{~mm}$ de diâmetro (Güss, África do Sul). Para a determinação dos teores de AT e SS, o suco foi extraído da secção equatorial de amostras de cinco frutos. O conteúdo 
de SS foi determinado usando-se um refratômetro digital com compensação automática da temperatura (Atago, Tókio). A AT foi determinada usando-se um titulador automático (Radiometer, Lyon, França) por meio da titulação de $2 \mathrm{~mL}$ de suco, diluídos em 20 $\mathrm{mL}$ de água, utilizando-se de solução de hidróxido de sódio $0,1 \mathrm{~N}$, até $\mathrm{pH} 8,1$. As ocorrências de pericarpo translúcido e podridões foram determinadas por análise visual de cada fruto e atribuição de notas (1 - ausência; 2 - presença).

Para a determinação das taxas respiratórias e de produção de etileno, amostras de frutos foram colocadas em jarras de $4 \mathrm{~L}$, supridas com ar comprimido, livre de etileno, com fluxo de $100 \mathrm{~mL} \mathrm{~min}^{-1}$. As concentrações de $\mathrm{CO}_{2}$ e etileno no ar efluente foram coletadas usando-se seringas e determinadas por cromatografia gasosa, conforme descrito em Argenta et al. (2003). As taxas respiratórias e de produção de etileno foram determinadas diariamente, nos frutos mantidos a $23^{\circ} \mathrm{C}$, após cada período de armazenamento, nos dois experimentos. Exceção ocorreu no experimento 2, após 150 dias de armazenamento sob $\mathrm{AC}$, quando não foi possível realizar estas determinações, e após 60 e 90 dias de armazenamento sob $\mathrm{AA}$, quando só foi possível realizar estas análises após $1 ; 6$ e 7 dias.

\section{Delineamento experimental e análise esta- tística dos dados}

Os experimentos foram conduzidos em delineamento inteiramente casualizado. Para as análises de firmeza da polpa e ocorrências de pericarpo translúcido e podridões, foram utilizadas 40 repetições, em que cada repetição correspondeu a um fruto. Vinte repetições foram analisadas após a remoção do armazenamento refrigerado e 20 repetições após 7 dias a $23^{\circ} \mathrm{C}$. Nas determinações dos teores de AT e SS, foram utilizadas quatro repetições, cada repetição com cinco frutos. As taxas respiratória e de produção de etileno foram determinadas com três repetições, cada repetição constituídas de 6 a 7 frutos. Os dados foram submetidos à análise de variância, e as médias de tratamentos, comparadas pelo teste de Fisher $(p<0,05)$, utilizando o programa SAS (SAS INSTITUTE, 2002).

\section{RESULTADOS E DISCUSSÃO}

Na colheita da safra de 2003, quivis 'Hayward' apresentaram firmeza da polpa de $15,9 \mathrm{lb}$, e o conteúdo de $\mathrm{SS}$, de $9,0 \%$ (experimento 1 ), enquanto na safra de 2004, a firmeza da polpa foi de $14,5 \mathrm{lb}$, e o conteúdo de SS, de 7,8\% (experimento 2).

No experimento 1, frutos tratados com 1-MCP apresentaram menor produção de etileno e maior conservação da firmeza da polpa durante todo o período experimental, não havendo diferenças entre as doses estudadas $\left(0,5\right.$ e $\left.1,0 \mu \mathrm{L} \mathrm{L}^{-1}\right)$ (Tabela 1$)$. Estes dados sugerem que a saturação das respostas de qui- vis 'Hayward' ao 1-MCP ocorre em dose $\leq 0,5 \mu \mathrm{L}$ $\mathrm{L}^{-1}$. Segundo Menniti et al. (2005), doses de 0,1 e $0,25 \mu \mathrm{L} \mathrm{L}^{-1}$ de 1-MCP são suficientes para retardar o amolecimento da polpa de quivi 'Hayward' durante o armazenamento refrigerado. Boquete et al. (2004) também observaram que o tratamento com 1-MCP $\left(0,5 \mu \mathrm{L} \mathrm{L}^{-1}\right)$ reduziu a produção de etileno em quivi 'Hayward', mesmo quando tratados após 30 dias sob armazenamento refrigerado. Kim et al. (2001) sugerem doses de 1-MCP superiores a $1,0 \mu \mathrm{L} \mathrm{L}^{-1}$ para maiores benefícios em quivis. Segundo estes autores, a presença dos pelos na superfície dos frutos pode dificultar a penetração do produto através da camada limítrofe. Em maçãs, a máxima eficiência do 1-MCP ocorre entre 0,5 e $1,0 \mu \mathrm{L} \mathrm{L}^{-1}$, podendo variar em função da cultivar e do tempo de armazenamento (WATKINS, 2006).

No experimento 2, o tratamento com 1-MCP também retardou o aumento da produção de etileno durante o período de exposição a $23^{\circ} \mathrm{C}$, após $60 ; 90 \mathrm{e}$ 120 dias de armazenamento sob AA (Figuras 1A, 1B e 1C). Este efeito do 1-MCP também foi observado em frutos armazenados sob AC, após 90 e 120 dias (Figuras 1E e 1F). A máxima taxa de produção de etileno foi observada em frutos não tratados com 1-MCP (controle), após 120 dias de armazenamento sob AA ou AC, seguido de 7 dias a $23^{\circ} \mathrm{C}$ (Figuras $1 \mathrm{C}$ e 1F). Segundo Arpaia et al. (1994), o quivi é considerado uma das espécies cujos frutos apresentam a maior sensibilidade ao etileno. Estes autores sugerem que concentrações de etileno inferiores a $10 \eta \mathrm{L} \mathrm{L}^{-1}$ na atmosfera de armazenamento são suficientes para acelerar o amadurecimento e ocasionar perdas na qualidade dos frutos. Neste trabalho, durante todo o período experimental, as concentrações de etileno no ambiente de armazenamento estiveram acima de $10 \eta \mathrm{L} \mathrm{L}^{-1}$, especialmente durante armazenamento sob AA. Considerando que o etileno tem papel crítico sobre o amadurecimento de quivis, a redução da taxa de produção de etileno evidencia o benefício do tratamento com 1-MCP (Tabela 1; Figuras 1A, 1B, 1C, 1E e 1F). Sob condição de AC, não foi verificado efeito significativo do tratamento com 1-MCP sobre a redução da taxa respiratória em ambos os experimentos (Tabela 1; Figuras 2D, 2E e $2 \mathrm{~F}$ ), embora ela tenha aumentado com o tempo de armazenamento. Os efeitos aditivos da baixa temperatura, redução da pressão parcial de $\mathrm{O}_{2}$, aumento da pressão parcial de $\mathrm{CO}_{2}$ e remoção do etileno do ambiente podem explicar, em parte, a ausência do benefício do tratamento com 1-MCP sobre a redução da taxa respiratória de frutos armazenados sob AC (WATKINS, 2006). Sob AA, o tratamento com 1-MCP reduziu, em relação ao tratamento-controle, a taxa respiratória durante o período a $23^{\circ} \mathrm{C}$, após 90 e 120 dias de armazenamento (Figuras 2B e 2C), o que não ocorreu após 60 dias de armazenamento (Figura 2A). Estes resultados evidenciam a efetividade do tratamento com 1-MCP na redução da taxa 
respiratória de quivis 'Hayward' armazenados sob AA. Resultados semelhante de respostas do 1-MCP sobre a taxa respiratória foram obtidos por Vieira et al. (2010) para quivis ‘Bruno' armazenados sob AA e AC em ambiente contendo etileno. Waclawovsky et al. (2003) também observaram benefícios do tratamento 1-MCP $\left(1,0 \mu \mathrm{L} \mathrm{L}^{-1}\right)$ sobre a redução da taxa respiratória em frutos armazenados sob $\mathrm{AA}$, na presença de $0,01 \mu \mathrm{L} \mathrm{L}^{-1}$ de etileno.

De forma similar ao observado no experimento 1 (Tabela 1), no experimento 2 , a redução da produção de etileno pelo tratamento com 1-MCP $\left(0,7 \mu \mathrm{L} \mathrm{L}^{-1}\right)$ foi associada ao aumento da conservação da firmeza da polpa de frutos armazenados sob AA e AC (Figuras 3A e 3D). A perda de firmeza da polpa em 60 dias de armazenamento sob AC, mais 7 dias a $23^{\circ} \mathrm{C}$, foi de $38 \%$ e $63 \%$ em frutos tratados e não tratados com 1-MCP, respectivamente (Figura 3D). Esses resultados evidenciam o efeito aditivo do tratamento com 1-MCP e da AC sobre o retardamento do amolecimento dos frutos. Já em frutos armazenados sob AA, a perda de firmeza da polpa em 30 dias, a $0^{\circ} \mathrm{C}$, mais 7 dias, a $23^{\circ} \mathrm{C}$, foi de $48 \%$ e $88 \%$ em frutos tratados e não tratados com 1-MCP, respectivamente (Figura 3A). Segundo Kim et al. (2001), os quivis amolecem rapidamente na presença de etileno exógeno. O rápido amolecimento da polpa verificado neste trabalho, em frutos armazenados sob AA, mesmo na presença de 1-MCP, pode ser atribuído à alta concentração de etileno no ambiente de armazenamento. Estes resultados estão de acordo com os observados por Brackmann et al. (1995). Estes autores também verificaram rápida perda da firmeza em quivis armazenados sob AA na presença de etileno. A eficiência do 1-MCP em retardar a taxa de perda da firmeza foi observada após 30 e 60 dias de armazenamento sob AA e AC, respectivamente. Após esses períodos, as taxas de redução da firmeza da polpa foram similares para frutos tratados e não tratados com 1-MCP (Figuras 3A e 3D). Segundo Kim et al. (2001), o fato de o 1-MCP efetivamente retardar a perda de firmeza apenas nas primeiras semanas de armazenamento sugere que a possível inibição da ação do etileno pelo produto em quivi é superada pela síntese de novos receptores para o etileno. Após este período, as diferenças entre frutos tratados e não tratados com 1-MCP refletem a manutenção de firmeza da polpa durante o período em que o produto esteve ativo. Crisosto e Garner (2001) também observaram perda do benefício do 1-MCP sobre a conservação da firmeza da polpa em quivi 'Hayward' após quatro semanas de armazenamento sob AA. Segundo Menniti et al. (2005) e Ilina et al. (2010), a aplicação de $0,1 \mu \mathrm{L} \mathrm{L}^{-1}$ de 1 -MCP logo após a colheita, e a reaplicação do 1-MCP, na mesma concentração, após a abertura da câmara, resulta em benefício adicional sobre o retardamento do amolecimento de quivi 'Hayward', quando comparado a somente uma aplicação.
A AT decresceu durante o período experimental para todos os tratamentos (Tabela 1; Figuras $3 \mathrm{~B}$ e $3 \mathrm{E}$ ). Os maiores decréscimos foram verificados em frutos não tratados com 1-MCP e armazenados sob AA. O tratamento com 1-MCP manteve a AT significativamente superior durante armazenamento sob AA, em relação a frutos não tratados (Figura 3B). Estes dados estão de acordo com os verificados por Waclawovsky et al. (2001). Já para frutos armazenados sob AC, em ambos os experimentos, o tratamento com 1-MCP manteve a AT sensivelmente superior em relação às de frutos não tratados, contudo estas diferenças não foram significativas (Tabela 1; Figura 3E). Exceção ocorreu no experimento 1, após 90 dias de armazenamento, no qual frutos tratados com 1-MCP, nas duas doses testadas, mantiveram AT significativamente superior à de frutos não tratados (Tabela 1). A ausência de benefício do 1-MCP sobre conservação da AT durante armazenamento sob AC (Figura 3E) coincide com a ausência do benefício do 1-MCP sobre redução das taxas respiratórias (Figuras $2 \mathrm{D}, 2 \mathrm{E}$ e $2 \mathrm{~F}$ ). Isto pode ser atribuído ao efeito sinérgico da redução da temperatura, alteração e controle das pressões parciais dos gases na atmosfera e da remoção do etileno do ambiente (WATKINS, 2006).

O amolecimento da polpa é considerado o principal indicador do fim da vida pós-colheita de quivi. No entanto, no ponto de consumo, os frutos devem apresentar firmeza da polpa entre 2 e $3 \mathrm{lb}$ (ARPAIA et al., 1994) e AT menor que $1,25 \%$ (VIEIRA et al., 2010). Considerando a firmeza da polpa de $4 \mathrm{lb}$ como limite inferior para classificação, embalagem e comercialização dos frutos, e $2 \mathrm{lb}$ correspondente ao limite de firmeza da polpa para consumo (ARPAIA et al., 1994), o tratamento com 1-MCP aumentou o potencial de armazenamento em aproximadamente 60 dias para frutos armazenados sob AA (Figura $3 \mathrm{~A}$ ), e em 30 dias para frutos armazenados sob AC (Figura 3D).

$\mathrm{O}$ conteúdo de SS aumentou durante o armazenamento sob AA e AC (Figuras 3C e 3F). Contudo, o tratamento com 1-MCP não teve influência sobre o acúmulo de SS, em ambas as condições de armazenamento e experimentos (Tabela 1; Figuras 3C e 3F). Estes dados estão de acordo com os verificados por Crisosto e Garner (2001), Waclawovsky et al. (2001) e Vieira et al. (2010).

O desenvolvimento de pericarpo translúcido aumentou durante o armazenamento sob AA. A incidência de pericarpo translúcido, após 90 e 120 dias de armazenamento sob AA, mais 1 ou 7 dias a $23^{\circ} \mathrm{C}$, foi significativamente menor quando os frutos foram tratados com 1-MCP (Tabela 2). Sob AC os frutos não desenvolveram índices significativos de pericarpo translúcido (Tabela 2). Estes resultados confirmam dados reportados por Arpaia et al. (1994). Estes autores observaram que a ocorrência de frutos com pericarpo translúcido aumenta com a duração do armazenamento e está associada ao amolecimento da 
polpa na presença de etileno. Os resultados obtidos mostram que o tratamento com 1-MCP em frutos armazenados sob AA e o armazenamento sob AC com remoção do etileno foram eficientes no controle do desenvolvimento de pericarpo translúcido, devido ao retardo na senescência pelo controle dos efeitos do etileno.

Não foi observado efeito significativo do tratamento com 1-MCP no controle de podridões em frutos armazenados sob AA e AC (dados não apresentados).

TABELA 1- Médias das taxas respiratórias, produção de etileno, firmeza de polpa, acidez titulável (AT) e sólidos solúveis (SS) em quivi 'Hayward' tratado logo após a colheita com diferentes doses de 1-MCP, e armazenados sob atmosfera controlada $\left(2,0 \mathrm{kPa}\right.$ de $\mathrm{O}_{2}+4,5 \mathrm{kPa}$ de $\mathrm{CO}_{2}$, a 0,0 $\pm 0,5^{\circ} \mathrm{C}, 92 \pm 3 \% \mathrm{UR}$, e com etileno no ambiente $\left.<40 \eta \mathrm{L} \mathrm{L}^{-1}\right)$, durante 90 e 120 dias, mais sete dias a $23^{\circ} \mathrm{C}$.

\begin{tabular}{lccccc}
\hline $\begin{array}{c}\text { Doses de 1-MCP } \\
\left(\mu \mathrm{L} \mathrm{L}^{-1}\right)\end{array}$ & $\begin{array}{c}\text { Etileno } \\
\left(\mathrm{nmol} \mathrm{kg}^{-1} \mathrm{~h}^{-1}\right)\end{array}$ & $\begin{array}{c}\text { Respiração } \\
\left(\mu \mathrm{mol} \mathrm{kg}^{-1} \mathrm{~h}^{-1}\right)\end{array}$ & Firmeza de polpa (lb) & $\begin{array}{c}\text { AT } \\
(\%)\end{array}$ & $\begin{array}{c}\text { SS } \\
(\%)\end{array}$ \\
\hline \multicolumn{7}{c}{90 dias sob AC } \\
0,0 & $395,0 \mathrm{a}$ & $280,3 \mathrm{a}$ & $2,0 \mathrm{a}$ & $1,16 \mathrm{a}$ & $15,0 \mathrm{a}$ \\
0,5 & $27,6 \mathrm{~b}$ & $234,7 \mathrm{a}$ & $7,5 \mathrm{~b}$ & $1,45 \mathrm{~b}$ & $14,8 \mathrm{a}$ \\
1,0 & $24,6 \mathrm{~b}$ & $266,0 \mathrm{a}$ & $6,2 \mathrm{~b}$ & $1,35 \mathrm{~b}$ & $14,8 \mathrm{a}$ \\
& & & 120 dias sob AC & & \\
0,0 & $374,8 \mathrm{a}$ & $507,9 \mathrm{a}$ & $1,9 \mathrm{a}$ & $1,14 \mathrm{a}$ & $15,2 \mathrm{a}$ \\
0,5 & $96,0 \mathrm{~b}$ & $441,8 \mathrm{a}$ & $5,6 \mathrm{~b}$ & $1,30 \mathrm{a}$ & $14,7 \mathrm{a}$ \\
1,0 & $185,0 \mathrm{~b}$ & $476,2 \mathrm{a}$ & $4,6 \mathrm{~b}$ & $1,22 \mathrm{a}$ & $14,5 \mathrm{a}$ \\
\hline
\end{tabular}

Médias seguidas pela mesma letra, na coluna, para cada período de armazenamento, não diferem pelo teste de Fischer $(\mathrm{p}<0,05)$.

TABELA 2 -Incidência de pericarpo translúcido (1-ausência; 2-presença) em quivi 'Hayward' tratado logo após a colheita com 0,0 (controle) e $0,7 \mu \mathrm{L}$ L-1 de 1 -MCP, e armazenados sob atmosfera ambiente $\left(21 \mathrm{kPa}\right.$ de $\mathrm{O} 2+0,03 \mathrm{kPa}$ de $\mathrm{CO} 2$, a $0,0 \pm 0,5^{\circ} \mathrm{C}, 90 \pm 3 \% \mathrm{UR}$, e com 200 a $250 \eta \mathrm{L} \mathrm{L}-1$ de etileno no ambiente, durante 30; 60; 90 e 120 dias, seguido de um ou sete dias a $23^{\circ} \mathrm{C}$ ), ou armazenados sob atmosfera controlada $\left(2,0 \mathrm{kPa} \mathrm{O}+4,5 \mathrm{kPa} \mathrm{CO}\right.$, a $0,0 \pm 0,5^{\circ} \mathrm{C}, 92 \pm 3 \% \mathrm{UR}$, e com etileno no ambiente menor que $40 \eta \mathrm{L}$ L-1, durante 60; 90; 120 e 150 dias, seguido de um ou sete dias a $23^{\circ} \mathrm{C}$ ).

\begin{tabular}{|c|c|c|c|c|c|c|c|c|}
\hline \multirow{3}{*}{$\begin{array}{c}\text { Dias a } \\
0^{\circ} \mathrm{C}\end{array}$} & \multicolumn{4}{|c|}{ Atmosfera ambiente (AA) } & \multicolumn{4}{|c|}{ Atmosfera controlada (AC) } \\
\hline & \multicolumn{2}{|c|}{1 dia a $23^{\circ} \mathrm{C}$} & \multicolumn{2}{|c|}{7 dias a $23^{\circ} \mathrm{C}$} & \multicolumn{2}{|c|}{1 dia a $23^{\circ} \mathrm{C}$} & \multicolumn{2}{|c|}{7 dias a $23^{\circ} \mathrm{C}$} \\
\hline & Controle & 1-MCP & Controle & 1-MCP & Controle & $1-\mathrm{MCP}$ & Controle & $1-\mathrm{MCP}$ \\
\hline 30 & $1,00 \mathrm{a}$ & $1,00 \mathrm{a}$ & $1,10 \mathrm{a}$ & $1,00 \mathrm{a}$ & & & & \\
\hline 60 & $1,00 \mathrm{a}$ & $1,00 \mathrm{a}$ & $1,05 \mathrm{a}$ & $1,00 \mathrm{a}$ & $1,00 \mathrm{a}$ & $1,00 \mathrm{a}$ & $1,00 \mathrm{a}$ & $1,00 \mathrm{a}$ \\
\hline 90 & $1,26 \mathrm{a}$ & $1,05 \mathrm{~b}$ & $1,20 \mathrm{a}$ & $1,05 \mathrm{~b}$ & $1,00 \mathrm{a}$ & $1,00 \mathrm{a}$ & $1,00 \mathrm{a}$ & $1,00 \mathrm{a}$ \\
\hline 120 & $1,31 \mathrm{a}$ & $1,12 \mathrm{~b}$ & $1,37 \mathrm{a}$ & $1,12 \mathrm{~b}$ & $1,00 \mathrm{a}$ & $1,00 \mathrm{a}$ & $1,00 \mathrm{a}$ & $1,00 \mathrm{a}$ \\
\hline 150 & & & & & $1,11 \mathrm{a}$ & $1,00 \mathrm{a}$ & $1,00 \mathrm{a}$ & $1,00 \mathrm{a}$ \\
\hline
\end{tabular}

Médias seguidas pela mesma letra na linha, comparando os tratamentos-controle e 1-MCP, não diferem pelo teste de Fischer (p<0,05). 


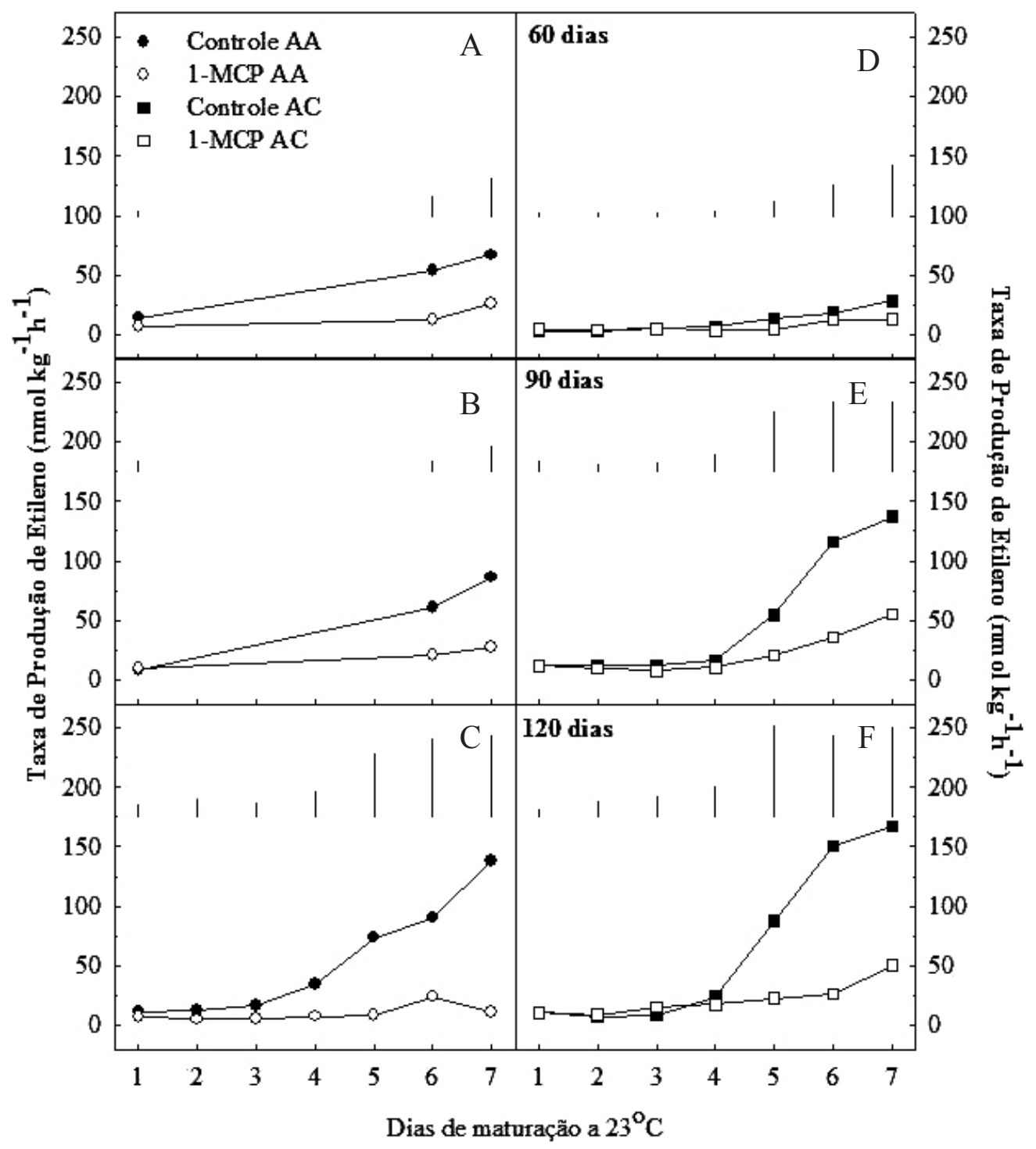

FIGURA 1- Taxas de produção de etileno em quivi 'Hayward' durante 7 dias a 23드. após 60; 90 e 120 dias de armazenamento sob atmosfera ambiente (AA), contendo $21 \mathrm{kPa}$ de $\mathrm{O}_{2}+0,03 \mathrm{kPa}$ de $\mathrm{CO}_{2}$, a $0,0 \pm 0,5^{\circ} \mathrm{C}, 90 \pm 3 \% \mathrm{UR}$, e com etileno no ambiente de 200 a $250 \eta \mathrm{L} \mathrm{L}^{-1}$, ou sob atmosfera controlada (AC), contendo $2,0 \mathrm{kPa}$ de $\mathrm{O}_{2}+4,5 \mathrm{kPa}$ de $\mathrm{CO}_{2}$, a $0,0 \pm 0,5^{\circ} \mathrm{C}, 92 \pm 3 \% \mathrm{UR}$, e com etileno no ambiente menor que $40 \eta \mathrm{L} \mathrm{L}^{-1}$. Os frutos foram tratados na colheita com 0,0 (controle) e $0,7 \mu \mathrm{L} \mathrm{L}^{-1}$ de 1 -MCP. As barras verticais, no interior de cada gráfico, representam as diferenças mínimas significativas entre tratamentos, calculadas pelo teste de Fischer $(p<0,05)$. 


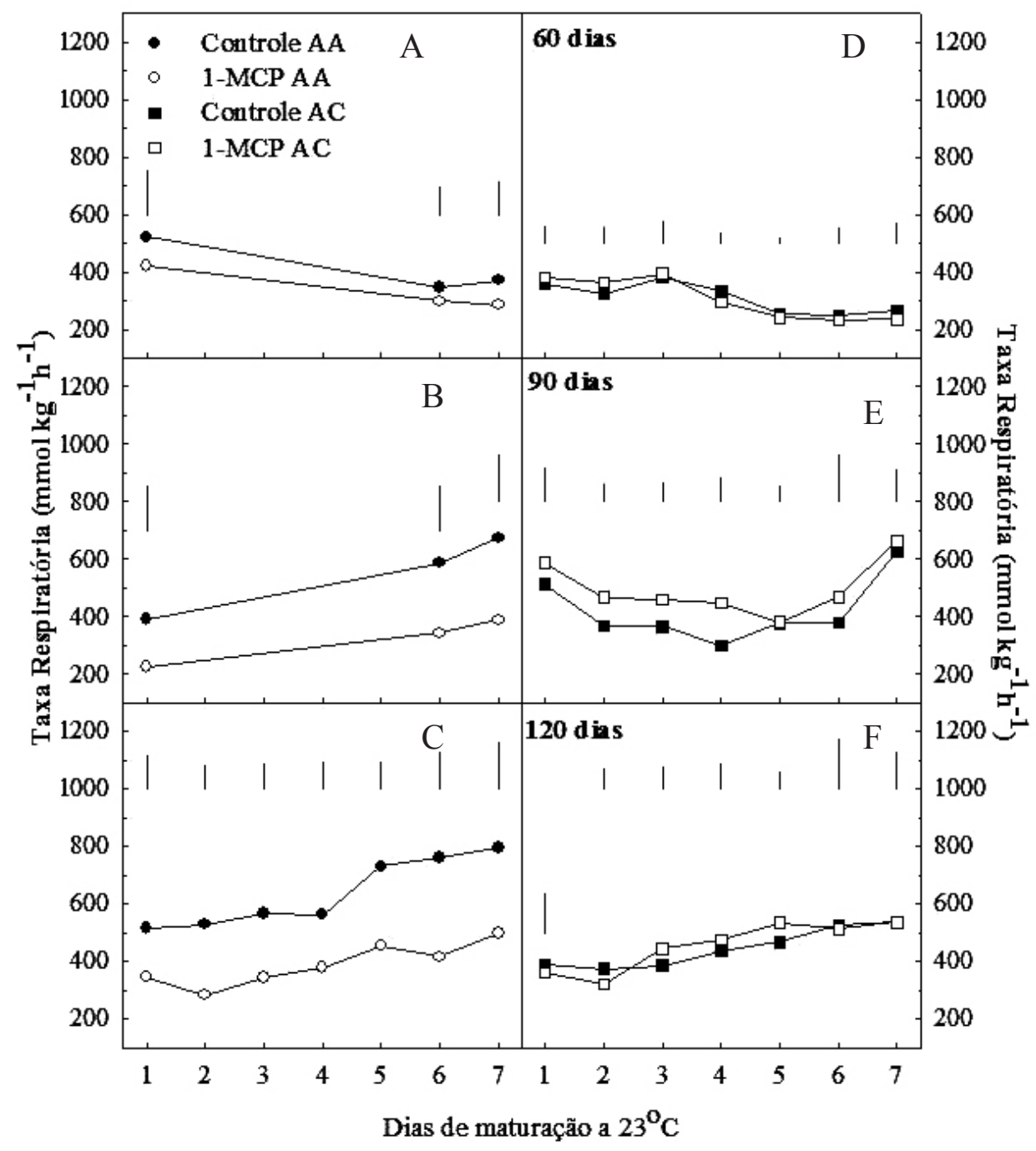

FIGURA 2-Taxas respiratórias em quivi 'Hayward' durante 7 dias a $23^{\circ} \mathrm{C}$, após $60 ; 90$ e 120 dias de armazenamento sob atmosfera ambiente (AA), contendo $21 \mathrm{kPa}$ de $\mathrm{O}_{2}+0,03 \mathrm{kPa}$ de $\mathrm{CO}_{2}$, a $0,0 \pm 0,5^{\circ} \mathrm{C}, 90 \pm 3 \% \mathrm{UR}$, e com 200 a $250 \eta \mathrm{L} \mathrm{L}^{-1}$ de etileno no ambiente, ou sob atmosfera controlada (AC), contendo $2,0 \mathrm{kPa}$ de $\mathrm{O}_{2}+4,5 \mathrm{kPa}$ de $\mathrm{CO}_{2}$, a $0,0 \pm 0,5^{\circ} \mathrm{C}, 92 \pm 3 \% \mathrm{UR}$, e com etileno no ambiente menor que $40 \eta \mathrm{L} \mathrm{L}^{-1}$. Os frutos foram tratados logo após a colheita com 0,0 (controle) e $0,7 \mu \mathrm{L} \mathrm{L}^{-1}$ de 1-MCP. As barras verticais, no interior de cada gráfico, representam as diferenças mínimas significativas entre tratamentos, calculadas pelo teste de Fischer $(\mathrm{p}<0,05)$. 


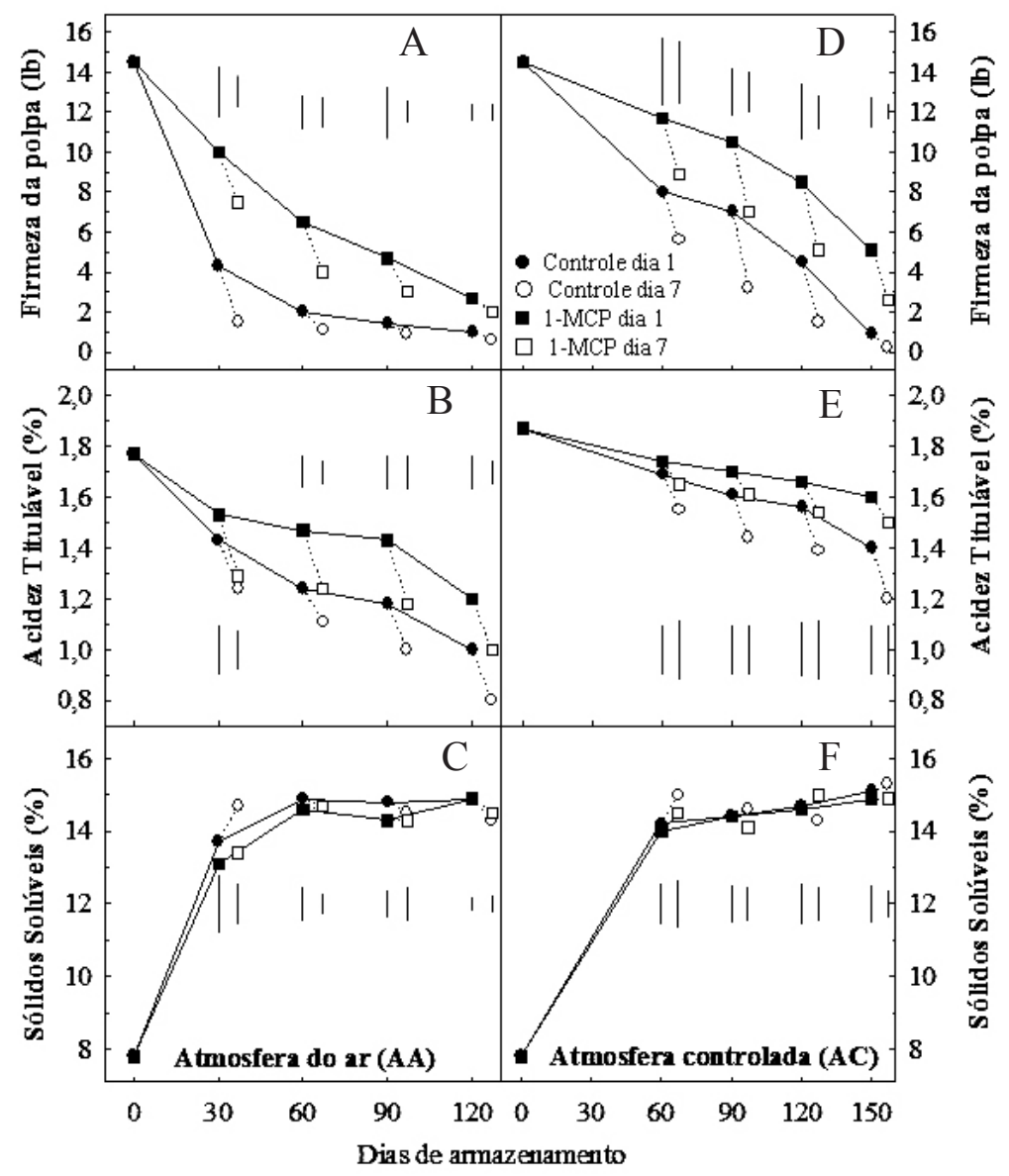

FIGURA 3-Firmeza da polpa (lb), acidez titulável (\%) e sólidos solúveis (\%) em quivi 'Hayward' armazenado sob atmosfera ambiente (AA), contendo $21 \mathrm{kPa}$ de $\mathrm{O}_{2}+0,03 \mathrm{kPa}$ de $\mathrm{CO}_{2}$, a $0,0 \pm 0,5^{\circ} \mathrm{C}$, $90 \pm 3 \%$ UR, e com 200 a $250 \eta \mathrm{L} \mathrm{L}^{-1}$ de etileno no ambiente, ou sob atmosfera controlada (AC), contendo $2,0 \mathrm{kPa}$ de $\mathrm{O}_{2}+4,5 \mathrm{kPa}$ de $\mathrm{CO}_{2}$, a $0,0 \pm 0,5^{\circ} \mathrm{C}, 92 \pm 3 \% \mathrm{UR}$, e com etileno no ambiente menor que $40 \eta \mathrm{L} \mathrm{L}^{-1}$, mais um ou sete dias de vida de prateleira a $23^{\circ} \mathrm{C}$. Os frutos foram tratados logo após a colheita com 0,0 (controle) e $0,7 \mu \mathrm{L} \mathrm{L}^{-1}$ de 1 -MCP. As barras verticais no interior de cada gráfico representam as diferenças mínimas significativas entre tratamentos, calculadas pelo teste de Fischer $(\mathrm{p}<0,05)$.

\section{CONCLUSÕES}

1-Em quivi 'Hayward' armazenado em atmosfera controlada (AC), tratamentos após a colheita com 1-MCP, nas doses de 0,5 e 1,0 $\mu \mathrm{L} \mathrm{L}^{-1}$, são igualmente eficientes no retardo do amadurecimento dos frutos.

2-O tratamento com 1-MCP $\left(0,7 \mu \mathrm{L} \mathrm{L}^{-1}\right)$ retarda o aumento na taxa de produção de etileno e proporciona maior conservação da firmeza da polpa em frutos armazenados sob atmosfera do ambiente (AA) e AC, e retarda o aumento na taxa respiratória e a queda na AT, e reduz a incidência de pericarpo translúcido em frutos armazenados sob AA.
3-O tratamento com 1-MCP $\left(0,7 \mu \mathrm{L} \mathrm{L}^{-1}\right)$ aumenta o potencial de armazenamento em aproximadamente 60 dias para frutos armazenados sob AA, e em 30 dias para frutos armazenados sob AC.

\section{AGRADECIMENTOS}

Ao CNPq e à CAPES, pela concessão de bolsas, e à AgroFresh Inc., pelo fornecimento do 1-MCP (EthylBloc $\left.{ }^{\mathrm{TM}}\right)$ 


\section{REFERÊNCIAS}

ARGENTA, L.C.; KRAMMES, J.G.; MEGGUER, C.A.; AMARANTE, C.V.T.; MATTHEIS, J. Ripening and quality of 'Laetitia' plums following harvest and cold storage as affected by inhibition of ethylene action. Pesquisa Agropecuária Brasileira, Brasília, v.38, n.10, p.1139-1148, 2003.

ARPAIA, M.L.; MITCHELL, F.G.; KADER, A.A. Postharvest physiology and causes of deterioration. In: HASEY, J.K.H.; JOHNSON, R.S.; GRANT, J.A.; REIL, W.O. Kiwifruit: growing and handling. Davis: University of California, 1994. p.88-93.

BLANKENSHIP, S.M.; DOLE, J.M. 1-Methylcyclopropene: a review. Postharvest Biology and Technology, Amsterdam, v.28, n.1, p.1-25, 2003.

BOQUETE, E.J.; TRINCHERO, G.D.; FRASCHINA, F.V.; SOZZI, G.O. Ripening of 'Hayward' kiwifruit treated with 1-methylcyclopropene after cold storage. Postharvest Biology and Technology, Amsterdam, v.32, n.1, p.57-65, 2004.

BRACKMANN, A.; SAQUET, A.A.; OSTER, A.H. Armazenamento refrigerado de kiwi em atmosfera normal e controlada. Revista Brasileira de Agrociência, Pelotas, v.1, n.2, p.107-111, 1995.

CRISOSTO, C.H.; GARNER, D. 1-MCP inhibits kiwifruit softening during storage. Perishables Handling Quarterly, Davis, v.108, p.19-20, 2001.
ILINA, N.; ALEM, H.J.; PAGANO, E.A.; SOZZI, G.O. Suppression of ethylene perception after exposure to cooling conditions delays the progress of softening in 'Hayward' kiwifruit. Postharvest Biology and Technology, Amsterdam, v.55, n.3, p.160-168, 2010.

KIM, H.O.; HEWETTI, E.W.; LALLU, N. Softening and ethylene production of kiwifruit reduced with 1-methylcyclopropene. Acta Horticulturae, The Hague, n.553, p.167-170, 2001.

MENNITI, A.M.; GREGORI, R.; DONATI, I. Effect of 1-methylcyclopropene on kiwifruit softening. Acta Horticulturae, The Hague, n.682, p.20952100, 2005 .

SAS INSTITUTE. Getting started with the SAS learning edition. Cary, 2002. 200p.

VIEIRA, M.J.; ARGENTA, L.C.; AMARANTE, C.V.T.; STEFFENS, C.A.; VIEIRA, A.M.F.D. Preservação da qualidade pós-colheita de kiwi 'Bruno' pelo controle do etileno. Revista Brasileira de Fruticultura, Jaboticabal, v.32, n.2, p.309-406, 2010.

WACLAWOVSKY, A.J.; DONAZZOLO, J.; NEUWALD, D.A.; BRACKMANN, A. Qualidade póscolheita de kiwis (actinidia deliciosa, Chevalier), cv. Bruno, tratados com 1-metilciclopropeno (1-MCP). In: CONGRESSO BRASILEIRO DE FISIOLOGIA VEGETAL, 8., 2001, Ilhéus. Anais... Ilhéus: SBFV, 2001. CD-ROM.

WATKINS, C.B. The use of 1-methylcyclopropene (1-MCP) on fruits and vegetables. Biotechnology Advances, Waterloo, v.24, n.4, p.389-409, 2006. 